\title{
Ceramide synthesis regulates biogenesis and packaging of exosomal MALAT1 from adipose derived stem cells, increases dermal fibroblast migration and mitochondrial function.
}

\section{Xiaoyuan Kong \\ VA James A Haley Hospital}

Niketa A. Patel

V.A. James A. Haley Hospital

Charles E. Chalfant

VA James A Haley Hospital

Denise R. Cooper ( $\sim$ dcooper@usf.edu )

Department of Veterans Affairs: US Department of Veterans Affairs https://orcid.org/0000-0001-52200192

\section{Research Article}

Keywords: Adipose-derived stem cells, MALAT1, Cell migration scratch assay, mitochondrial stress test, Ceramide, sphingomyelinase, Exosomes, Extracellular vesicles, Human dermal fibroblasts, ncRNA

Posted Date: November 30th, 2021

DOI: https://doi.org/10.21203/rs.3.rs-1040924/v1

License: (c) (i) This work is licensed under a Creative Commons Attribution 4.0 International License.

Read Full License 


\section{Abstract \\ Background}

The function of exosomes, small extracellular vesicles (EVs) secreted from human adipose-derived stem cells (ADSC), is becoming increasingly recognized as a means of transferring the regenerative power of stem cells to injured cells in wound healing. Exosomes are rich in ceramides and long noncoding RNA (IncRNA) like metastasis-associated lung adenocarcinoma transcript 1 (MALAT1). We identified putative ceramide responsive cis-elements (CRCE) in MALAT1. We hypothesized that CRCE respond to cellular ceramide levels to regulate EVs MALAT1 packaging. MALAT1 levels by many cells exceeds those of protein coding genes and it's expression is equally high in exosomes. Ceramide also regulates exosome synthesis, however, the contents of exosome cargo via sphingomyelinase and ceramide synthase pathways has not been demonstrated.

\section{Methods}

ADSC were treated with an inhibitor of sphingomyelinase, GW4869, and stimulators of ceramide synthesis, C2- and C6-short chain ceramides, prior to collection of conditioned media (CM). EV were isolated from $\mathrm{CM}$, and then used to treat human dermal fibroblast (HDF) cultures in cell migration scratch assays, and mitochondrial stress tests to evaluate oxygen consumption rates (OCR).

\section{Results}

Inhibition of sphingomyelinase by treatment of ADSC with GW4869 lowered levels of MALAT1 in small EVs. Stimulation of ceramide synthesis using C2- and C6- ceramides increased cellular, EVs levels of MALAT1. The functional role of EV MALAT1 was evaluated in HDF by applying EVs to HDF. Control EVs increased migration of HDF, and significantly increased ATP production, basal and maximal respiration OCR. EV from GW4869-treated ADSC inhibited cell migration and maximal respiration. However, EV from C2- and C6-treated cells, respectively, increased both functions but not significantly above control EV except for maximal respiration. EV were exosomes except when ADSC were treated with GW4869 and C6ceramide, then they were larger and considered microvesicles.

\section{Conclusions}

Ceramide synthesis regulates MALAT1 EV content. Sphingomyelinase inhibition blocked MALAT1 from being secreted from ADSC EVs. Our report is consistent with those of MALAT1 increasing cell migration and mitochondrial MALAT1 altering maximal respiration in cells. Since MALAT1 is important for exosome function, it stands that increased exosomal MALAT1 should be beneficial for wound healing as shown with these assays. 


\section{Background}

Exosomes are ceramide-enriched small extracellular vesicles generated by inward budding of the endosomal membrane and secreted when the multivesicular endosomes fuse with the plasma membrane ${ }^{1,2}$. These small vesicles, 40-160 nm, contain a cargo of protein factors, mRNA, long noncoding RNA (IncRNA), snRNA, tRNA and microRNA (miRNA) in addition to lipids, that are taken up by other cells to mediate intercellular communication ${ }^{3}$. Although miRNAs in exosomes have been widely investigated, IncRNA packaging and function in these vesicles is largely unknown. The IncRNA, metatasis-associated lung adenocarcinoma transcript 1 or MALAT1 is abundantly expressed at levels that are even higher than many protein coding genes, including $\beta$-actin or GAPDH by cells ${ }^{4}$. MALAT1 is processed into a 6.7 or $8.4 \mathrm{~kb}$ transcript from mice and humans ${ }^{5}$. We have shown that MALAT1 is one of the most abundant IncRNA identified in exosomes from human adipose derived stem cells (ADSC) ${ }^{6}$. Exosomes, when applied to various models of wound healing, increase the rate of cell migration in scratch assays and an in vivo model of ischemic wounds ${ }^{7}$. MALAT1 has numerous cellular functions including alternative splicing of pre-mRNA, control of gene expression, recruitment of transcription factors, and recruitment of competitive endogenous RNAs and miRNA 4 . MALAT1 can bind serine/arginine-rich (SR) splicing factors (SF) such as SRSF1 to promote cell proliferation ${ }^{8}$. In the case of cultured ADSC, most of the MALAT1 detected is exosomal suggesting that in stem cells it functions differently from its nuclear splicing action in differentiating cells ${ }^{9}$. When hADSC begin to differentiate to adipocytes, high levels of exosomal MALAT1 are no longer the case, and most MALAT1 is retained by preadipocytes and adipocytes. We wanted to determine how MALAT1 was destined for packaging into ADSC exosomes.

Exosomes are rich in ceramide ${ }^{10}$, and MALAT1 contains multiple ceramide response cis-elements (CRCE) core motifs within it ${ }^{11}$. CRCE in RNA can regulate transcription, alternative splicing of transcripts, transport of proteins into vesicles, activity of enzymes, and secretion of exosomes ${ }^{11-14}$. We hypothesized that ceramide production played a role in the packaging and secretion of MALAT1. Exosome biogenesis is regulated in a ceramide-dependent manner dependent upon neutral sphingomyelinase (n-SMase) ${ }^{10}$. GW4869, an inhibitor of n-SMase1 and 2 reduces the release of proteolipid protein (PLP)-positive exosomes ${ }^{2}$. In the presence of GW4869, exosomes are not secreted but microvesicles, $>300 \mathrm{~nm}$, are detected instead. Here, we report the effects of GW4869 treatment on exosomes and microvesicle function in cells. C2- and C6-ceramide incorporate into longer species of ceramide via de novo ceramide synthesis. This effect was investigated on MALAT1 levels in cells and exosomes and microvesicles, and on cell migration and mitochondrial function of human dermal fibroblasts (HDF) as a model for wound healing. Our results indicate that the loss and gain of MALAT1 levels in exosomes and microvesicles via ceramide synthesis has functional consequences for cell migration and mitochondrial function related to wound healing.

\section{Materials And Methods}




\section{Culture of adipose derived stem cells:}

Human adipose derived stem cells (hADSC) were purchase from ZenBio, Inc (Item \# ASC-F). The cells screened negative for HIV-1, HIV-2, HTLC-1, HTLV-2, Hep-B, Hep-C, and mycoplasma. HADSC were isolated from subcutaneous adipose tissue of normal, nondiabetic donors between 25 and 45 years of age undergoing elective surgery and with $\mathrm{BMI}<23.3$. Cells were cultured according to the manufacturer's instructions.

\section{Collection of conditioned media (CM):}

Exosomes and microvesicles were isolated from conditioned media (CM). For CM collection, hADSC were grown to $90 \%$ confluence in T75 flasks, media was replaced with serum-free mesenchymal stem cell basal media, with StemFlex medium kit (Gibco, Cat\# A33494-01) and CM was collected after 48 hours.

For GW4869-CM, the hADSC cells were treated with GW $4869(1 \mu \mathrm{M})$ (from Cayman Chemical Item No\# 13127) in serum-free media for 48 hours. For C2-ceramide CM, hADSC were treated with C2-ceramide (Cayman Chemical Item No\# 62510), a biologically active, cell permeable and less hydrophobic analog of natural ceramide, at $20 \mu \mathrm{M}$ in serum-free media, $48 \mathrm{hrs}$. For C6-ceramide CM, hADSC were treated with C6-ceramide (Cayman Chemical Item No\# 62525) at $20 \mu \mathrm{M}$ with serum-free media, $48 \mathrm{hrs}$.

\section{Isolation of exosomes and microvesicles:}

Exosomes and microvesicles were isolated using Exo-Spin Exosome Purification Kit (Cell Guidance System, Cat\# EX01-25). Briefly, $45 \mathrm{ml}$ of CM was transferred to a conical tube and centrifuged at $300 \mathrm{xg}$ for $10 \mathrm{~min}$ to remove any unattached cells. The supernatant was transferred to a new tube and spun at $16,000 \mathrm{x} g$ for $30 \mathrm{~min}$ to remove any remaining cell debris. Next, the supernatant was transferred to a new tube, and $1 / 2$ volume of Exo-Spin Buffer was mixed with it and incubated at $4^{\circ} \mathrm{C}$ overnight. This was then centrifuged at $16,000 \times \mathrm{g}$ for 1 hour, carefully aspirated and the supernatant was discarded. The exosome-containing pellet was resuspended in $100 \mu \mathrm{l}$ of PBS. The Exo-Spin Column was prepared following manufacturer's instructions. The exosome containing pellet $(100 \mu \mathrm{l})$ was carefully applied to the top of column and the column was placed into the waste tube. This was then centrifuged at $50 \times \mathrm{g}$ for one min, and the eluate was discarded. The column was then placed into a $1.5 \mathrm{ml}$ microcentrifuge collection tube, and $200 \mu \mathrm{l}$ of PBS was applied to the column. This was centrifuged at $50 \mathrm{xg}$ for one min. The $200 \mu$ eluate contained the purified small extracellular vesicles containing exosomes and microvesicles.

\section{Measurement of MALAT1 in cells and small extracellular vesicles (sEV):}

Human ADSC were cultured in T-75 flasks. For CM collection, hADSC were grown to about $90 \%$ confluence, medium was replaced with serum-free mesenchymal stem cell basal medium, we used StemFlex medium kit (Gibco, Cat\# A33494-01) and CM (15 ml) were collected after 48 hours. Total cellular RNA was isolated from sEV using RNeasy Mini Kit (Qiagen, Germantown, MD). One microgram of 
total RNA was used to synthesize complementary cDNA with iScript cDNA Synthesis Kit (Cat \# 1708891, Bio-Rad, Hercules, CA) according to the manufacturer's protocol.

The Real-Time PCR reaction was performed using $2 \mu$ of cDNA with $1 \mu$ l of 20X Taqman Human MALAT1 primer/probe set (Assay ID number Hs00273907_S1), (Applied Biosystems, Foster City, CA), $10 \mu \mathrm{L}$ of TaqMan® Fast Advanced Master Mix (Applied Biosystems, Foster City, CA) and $7 \mu \mathrm{L}$ of Nuclease-Free Water for a total volume of $20 \mathrm{uL}$. As an internal control, a second Real-Time PCR reaction was performed using $2 \mathrm{uL}$ of cDNA with $1 \mu \mathrm{L}$ 20X Taqman Human GAPDH primer/probe set(Assay ID number Hs03929097_g1), (Applied Biosystems, Foster City, CA). Thermocycling conditions were as follows: $50^{\circ} \mathrm{C}, 2 \mathrm{~min} ; 95^{\circ} \mathrm{C}, 10 \mathrm{~min}$; and 40 cycles of $95^{\circ} \mathrm{C}$ for $15 \mathrm{~s}$ and $60^{\circ} \mathrm{C}$ for 1 min using Applied BioSystems ViiA 7 System. The Human MALAT1 mRNA Relative expression (RQ) to GAPDH was calculated. Each sample was run in duplicate. SEV RNA was isolated as described above. Human MALAT1 mRNA relative expression (RQ) to GAPDH was calculated. Each sample was run in duplicate.

\section{HDF scratch assays:}

Human Dermal Fibroblasts-adult (HDF-a) cells were purchased from ScienCell (Catalog N0. 2320). HDF-a were from adult human skin and were negative for HIV-1, HBV, HCV, mycoplasma, bacteria, yeast and fungi. Cells were subcultured in Fibroblast Medium (FM, Catalog N0\# 2301) until approximately 70\% confluent and media changed every other day until the cells were about $85 \%$ confluent.

For performing a wound healing scratch assay, we used the Ibidi Culture-insert 2 well (Ibidi, Catalog N0. 80209). Use sterile tweezers for transfer, the sterile 2-well insert was placed into each well of a 12-well plate, The HDF-a cell suspension of about $3.6 \times 10^{5} / \mathrm{ml}$, was added $(70 \mu$ l of cell suspension) to each well of the plate with inserts. Cells were cultured at $37^{\circ} \mathrm{C}$ with $5 \% \mathrm{CO}_{2}$ as usual. After 24 hours cells were confluent, inserts were removed, and the plate was rinsed once with PBS to remove cell debris. Then cells were preincubated with mitomycin $\mathrm{C}(10 \mu \mathrm{g} / \mathrm{ml})$ for 2 hours to block further proliferation so that only migration was followed.

Isolated exosomes $20 \mu \mathrm{g} / \mathrm{ml}$ (protein) were then added to wells. Basic fibroblast growth factor (bFGF, Gibco, Cat \# 13256-029) was used as the positive control, $20 \mathrm{ng} / \mathrm{ml}$. Wound healing assay photo images were acquired at $0,8,26$, and 48 hours. Images were analyzed by Ibidi Metavi Wound Healing Analysis Automatic Cellular Analysis System and by counting cells that had migrated into the scratched field. Data from each time point and treatment were analyzed by two-tailed ANOVA with $p<0.05$.

\section{Mitochondrial stress test:}

Human Dermal Fibroblasts-adult (HDF-a) cells were purchased from ScienCell (Catalog N0. 2320) and cultured in a T-75 flask following the manufacture's instructions. For the Mitochondrial Stress test the HDF-a, $5.5-6 \times 10^{3}$ cells, were plated into wells of a poly-L-lysine coated XFp cell culture miniplate with 80 $\mu \mathrm{l}$ of growth medium. In two of the 8 wells, no cells were plated for background correction. Sterile water $(400 \mu \mathrm{l})$ was added to each chamber of the moat. Cells grew overnight in a cell culture incubator. Cells 
were then treated with exosomes prior to the stress test. Exosomes $(20 \mu \mathrm{g}$ protein $/ \mathrm{ml})$ were added to the wells with serum free medium, and cultured in a $\mathrm{CO}_{2}$ incubator for 2-24 hours. The Seahorse Mito Stress Test used the Agilent Seahorse XFp Extracellular Flux Analyzer. Oxygen consumption rate (OCR) was measured using the mitochondrial stress test kit (Cat \# 103010-100) following instructions. Agilent Seahorse XF DMEM media (Part \# 103575-100) containing $10 \mathrm{mM}$ glucose, 2 mM L-glutamine, and 1 $\mathrm{mM}$ sodium pyruvate were used prior to the assay and the compounds for loading the sensor cartridge ports for the Agilent Seahorse XFp analyzer were: $1.0 \mu \mathrm{M}$ of Oligomycin (Port A), $1 \mu \mathrm{M}$ of FCCP (Port B) and $0.5 \mu \mathrm{M}$ of Rotenone/Antimycin A (Port C).

The Agilent Seahorse XF cell Mito Stress Test report generator automatically calculated the Agilent Seahorse XFp Cell Mito Stress Test parameters from WAVE data that was exported to Excel. Exosome preparations were tested in 3-4 separate assays. Significance was tested using a two-tailed Student's $t$ test (Microsoft Excel) with at least three independent biological replicates (n), with the Mann-Whitney $U$ test or with one-way ANOVA as indicated (GraphPad Prism) $\left({ }^{\star} p<0.05,{ }^{* \star} p<0.01\right.$, and $\left.{ }^{\star \star *} p<0.001\right)$.

\section{SEV size and charge:}

SEV size was determined using Malvern Nano Series Zetasizer Nano ZS90. A SEV aliquot of $50 \mu \mathrm{l}$ was added to $450 \mu$ l nuclease-free water and mixed in a cuvette for determining the size of SEV in the instrument.

\section{Results}

\section{Regulation of ceramide synthesis alters MALAT1 levels in ADSC and SEVj}

MALAT1 is the most abundant IncRNA in exosomes secreted by hADSC ${ }^{9}$. Since the levels of exosomal MALAT1 detected by qPCR are greater than that detected in the cells themselves, we questioned how MALAT1 was recruited and packaged into exosomal vesicles. Sphingomyelin (SM) is hydrolyzed into phosphorylcholine and ceramide (Cer) by a family of sphingomyelinases that function at acidic, neutral, or alkaline $\mathrm{pH}^{15}$. Sphingomyelinases are specifically associated with cellular compartments ${ }^{16}$. Previous studies showed that SM hydrolysis and Cer formation participates in endosomal sorting complex required for transport (EXCRT)-independent biogenesis of intraluminal vesicles inside MVBs, the vesicles that are released as exosomes upon MVB fusion with the plasma membrane ${ }^{2}$. Those studies showed that total Cer was enriched by $>3$-fold in a subpopulation of exosomes containing proteolipid protein (PLP). It was proposed a lipid based mechanism for exosome formation was involved. Hence, we examined release of exosomes here by treating stem cells with GW4869, an inhibitor of n-SMase 1 and nSMase 2 which increase long chain ceramides. Exosomal packaging of MALAT1 was demonstrated using GW4869 treated stem cells to probe whether the inhibition of ceramide synthesis altered sEV MALAT1 mRNA levels ${ }^{17}$. Human ADSC were treated with GW4869 for 48 hrs while sEV were collected in serum free media to provide conditioned media (CM). total RNA from hADSC, exosomes and 
microvesicles were isolated and analyzed for MALAT1 by qPCR. All preparations of exosomes and microvesicles exhibited the same level of CD63, an exosomal surface marker (Fig. 1A). Exosomes and microvesicles were quantified by protein content which we showed earlier to be related to a profile of sEV as detected by light scattering. Figure $1 \mathrm{~b}$ demonstrates that MALAT1 concentrations in the hADSCs were reduced $75 \%$ by GW4869 compared to control, untreated hADSC. SEV MALAT1 content was also significantly diminished by treating the hADSC with the nSMNase inhibitor during collection of CM (Fig. 1c).

Our finding is novel in that ceramide synthesis appears to be involved in the generation and packaging of MALAT. This could be via a putative CRCE in its promoter as predicted by bioinformatics. The longest MALAT1 transcript from human is 8708 nt in length [GenBank: NR_002819.2], and the transcriptional start site was defined ${ }^{18}$. MALAT1 also contains several CRCEs core motifs within its sequence as shown in Table 1. A core motif is defined as the sequence within the RNA recognition motif that are found in RNA binding proteins controlling the production, maturation, localization, translation, and degradation of cellular RNAs. Here, we did not identify the RNA binding domain protein (RBD), but inspected the MALAT1 sequence for motifs resembling those described previously for a ceramide responsive RBD protein ${ }^{11}$. Although we did not identify a full-length motif as previously reported, we were able to identify seven motifs with six to nine base matches. Most RBDs make contact with 3-5 contiguous RNA bases ${ }^{19}$. This number of motifs were not found in GAS5, another IncRNA in exosomes or in HOTAIR, a IncRNA also identified in $\mathrm{CM}^{6}$.

\section{C2- and C6-ceramide treatment of hADSC increases MALAT1 levels.}

To further define ceramide function in MALAT1 exosomal packaging, we treated ADSC with C2- and C6ceramides during the $48 \mathrm{hr}$ collection of $\mathrm{CM}$. These precursors are capable of being incorporated into long chain ceramides by several ceramide synthase pathways. C2-ceramide, which stimulates the de novo pathway of ceramide synthesis, increased the cellular amount of MALAT1 by $30 \%$ compared to control cells (Figure 1b), but increased exosomal MALAT1 by 3-fold compared to control exosomes (Figure 1c). These sEVs however, were larger in size than exosomes and considered to be microvesicles, corresponding in size to those from GW4869 treated cells (Table 2). For comparison, C6-ceramide was also used to treat cells. It is known to incorporate into different ceramide synthase pathways than $\mathrm{C} 2$ ceramide. C6-ceramide increased cellular MALAT1 3.5-fold and exosomal MALAT1 content by 4.5-fold. The size of the SEV corresponded to exosomes (Table 2). C6-ceramide treated cells increased both cellular and secreted MALAT1. This indicates that potentially two ceramide synthase pathways are involved in MALAT1 transcription, cellular retention and packaging into exosomes. Hence, ceramide synthesis increased packaging of MALAT1 in proportion to its intracellular levels.

Human dermal fibroblast migration responded to ceramide and MALAT1 containing exosomes in scratch assays. 
Given that ceramide synthesis modulated packaging of MALAT1, we examined sEV and exosomal function on cellular migration by treating HDF with the different $s E V$ isolates using a modified scratch assay where myriocin $\mathrm{C}$ pre-treatment blocked cell proliferation so that only migration speed was observed. We noted that control exosomes increased cell migration as we had previously reported (Figure 2a) and microvesicles from GW4869-treated cells, containing significantly lower levels of MALAT1, slowed cell migration compared to control exosomes. However, migration resulting from mirovesicles collected from C2-treated stem cells stimulated migration even more than the control exosomes but less than $\mathrm{C} 6$ treated cell derived exosomes containing higher levels of MALAT1 (Fig. 2a,b). This would indicate that the larger microvesicles could also be therapeutic if they contained MALAT1. The increased levels of MALAT1 in microvesicles (4- to 5 - fold over control exosomes) did not increase cell migration in a dose response manner however, but migration was significant as shown in Figure $2 \mathrm{~b}$. This suggests that there is an optimal level of MALAT1 that is utilized by cells to increase HDF migration.

\section{Exosome treatment of HDF improves mitochondrial function.}

One aspect of exosomes and microvesicles that has not been investigated is their potential for increasing mitochondrial function. Increased cellular ATP is required for wound healing, and the fine tuning of reactive oxygen species (ROS) is integral to this process. We studied the effect of sEV from GW4869treated hADSC (GW4869 sEV) on HDF subjected to the Mitochondrial Stress Test. These microvesicles, with very little MALAT1, resulted in decreased OCR ( $\mathrm{pmol} / \mathrm{min} / 5,500$ cells) detected as ATP production by $40 \%$ and also decreased basal and maximal respiration (Fig. 3a).

In another Mitochondrial Stress Test, control, untreated exosomes increased HDF basal respiration (OCR) by $45 \%$ compared to control (no exosomes) HDF (Fig. 3a). There were no significant differences between control exosomes and C2- or C6- exosomes or microvesicles. The overall maximal respiration from control and C2- and C6- isolated microvesicles/exosomes was increased 50\% with a significant differences between control and Cer treated exosomes for ATP production. C2-and C6-ceramide microvesicles and exosomes contained more MALAT1 as shown in Fig. 1c. The inclusion of more MALAT1 in C2- and C6- exosomes did not significantly alter ATP production or basal respiration more than the control exosomes suggesting that MALAT1 was important for increasing parameters related to mitochondrial reactive oxygen species (ROS).

Here, the first measurement is the basal oxygen consumption rate (OCR) measured in cells prior to injection of oligomycin (Fig. 3b). The exposure to oligomycin, the ATP synthase inhibitor revealed that control exosomes increased ATP synthesis and GW4869 microvesicles reduced ATP. The increased MALAT1 in C2- and C6-microvesicles/exosomes also increased ATP production (Fig. 3a), but not significantly from control exosomes. Maximal respiration, calculated after FCCP addition and Antimycin A + Rotenone was increased by control exosomes, C2- microvesicles, and C6- exosomes (Fig. 3a, 3b). The GW4869 microvesicles blocked maximal respiration when compared to control exosomes (Fig. 3a, 3c). This suggests that maximal OCR estimated by FCCP compared to basal respiration demonstrated mitochondria using less than the maximal rate of electron transport that can be supported by substrate 
from the cells. The difference between basal and maximal respiration is the spare or reserve capacity of energy. The reserve is depleted under conditions of pressure overload or ischemia (Fig. 3a). Very often it can be depleted. Whether the HDF utilize the maximal electron transport activity for ATP syntheses depends on the capacity of the oxidative phosphorylation system which may limit a response. The reserve component capacity is cell dependent and linked to bioenergetic health whether ATP synthesis or other functions occur. In the spare receptor capacity, we see the difference between C2- and C6- sEV and project that two ceramide synthase pathways alter other components of the sEV. C2-treatment increased the size of the sEV and they were essentially the same size as those from GW4896-treated cells. All sEV preparations had negative charges as expected (data not shown).

\section{Discussion}

The packaging of IncRNAs such as MALAT1 with at least 7 partial or core motif ceramide response elements was regulated by the C2-and C6-sphingomyeliase pathways when stem cells were exposed to exogenous short chain ceramides. C2-treatment altered the size of the sEV, but C6-treatment did not. These sEV, or exosomes, increased the migration of HDF in scratch assays, and also increased HDF maximal respiration by $40-50 \%$ in mitochondrial stress assays. Increased mitochondrial function is necessary for the healing of dermal wounds as represented by the scratch assay.

Overall, the packaging of MALAT1 into ADSC exosomes and microvesicles appears to be regulated by three ceramide synthase pathways in different manners. A loss of function in a sub population of exosomes treated with siRNA for MALAT1 decreased the ability of cells to migrate in initial studies ${ }^{9}$. However, MALAT1, increased up to 4.5-fold in C6-treated exosomes as determined by comparison to control exosomes, did not further improve dermal fibroblast cell migration, basal cellular respiration and ATP production. This upper limit of MALAT1 function may be due to a protective mechanism from too much of a IncRNA that is often associated with cancer ${ }^{20}$. It may also be due to a saturating level intracellularly.

The finding that overexpression of MALAT1 via ceramide synthase pathways is important for wound healing is novel. Sphingolipids are known to exert critical roles in disease states. Mitochondrial ceramide generation and transport of ceramide to mitochondria regulate its function as shown by the increase in exosome function from ceramide and MALAT1 enriched exosomes ${ }^{21}$.

In our study, exogenous C2- and C6-derived ceramides altered maximal mitochondrial respiration of HDF exposed to microvesicles and exosomes. This effect of $\mathrm{C} 2$-derived ceramide was shown to alter gene expression by inhibition of ROS production in invasive cells via the inhibition of matrix metalloproteinase gene expression ${ }^{21}$. However, maximal respiration is also associated with modulation of ROS and this aids in wound healing via fibroblast mitochondrial affecting genes central to wound healing ${ }^{22}$. Our results show that C6-derived exosomes promoted a significant increase in cell migration at later time points. C2-derived microvesicle application also demonstrated a higher rate of scratch closure, but not 
significantly different from control exosomes which may indicate specificity for exosomes in wound healing vs sEV.

The fact that C2- and C6-derived ceramides both increased MALAT1 levels is not surprising, as MALAT1 contains a ceramide responsive element in its promoter sequence determined by bioinformatics.

Ceramide is generated by three metabolic pathways: de novo synthesis, sphingomyelin hydrolysis, and the salvage pathway ${ }^{23}$. The fact that the inhibitor of sphingomyelin hydrolysis, GW4869 blocked MALAT1 transcription, packaging and secretion suggests a pivotal role for ceramides in packaging of sEV IncRNA cargo. C2- and C6-ceramide treatment of cells is known to increase long chain (14-26 carbons) ceramide concentrations via a set of six ceramide synthases with some having overlapping specificity and others synthesizing C18 ceramide, C18-22 ceramide and C22-24 ceramide while one yields C26-ceramide and above. The salvage pathway produces ceramide from catabolism of complex sphingolipids yielding sphingosine ${ }^{23}$.

Ceramide synthases are cell/tissue specific, and usually found in the endoplasmic reticulum, but also in mitochondria where they are components of inner and outer mitochondrial membranes. C2-ceramide stimulates mitochondrial complex IV activity ${ }^{24}$. Other ceramides inhibit complex $\mathrm{II}^{25}$. The effects of different ceramides has varying effects on mitochondrial membrane potential. C6-ceramide caused mitochondrial depolarization ${ }^{26}$. Hence, the effects of the C2-ceramide precursors could have effects on sEV size that is transferred to the HDF in addition to MALAT1 which may carry ceramides into sEV and subsequently transfer it to HDF.

Our result of MALAT1 laden exosomes increasing basal respiration represents a number of options for cell migration and mitochondrial function which are both stimulated or inhibited by these pathways when packaged into exosomes. MALAT1 was found in the mitochondria of HepG2 cells ${ }^{27,28}$. However, in these studies MALAT1 originated in the nucleus and was found enriched in mitochondria where it interacted with mitochondrial proteins. Our study suggests that exosomal MALAT1 could be utilized by HDF mitochondria to alter their function.

The downregulation of MALAT1 exosomal content by GW4896 treatment of stem cells as shown here resulted in the inhibition of mitochondrial ATP production, basal and maximal respiration. Whether the effect was due to MALAT1 depletion, the size of the sEV, or other factors altered by inhibition of ceramide synthesis is unknown. However, the role of MALAT1 in mitochondrial function was established previously, and our results demonstrate a role for the IncRNA in wound healing and mitochondrial function of noncancerous cells.

Studies of MALAT1 function often focus on the overexpression of the IncRNA in cancer cells. Here, we are focusing on the function of this abundant non-coding RNA when packaged into exosomes derived from healthy stem cells. The finding that MALAT1 contains ceramide response elements explains how the ceramide synthesis pathways regulate its transcription and packaging via ceramide dependent activities. 
The function of a putative CRCE in IncRNA has not been reported. The original element contained 15 nucleotides. Here, we have found putative core elements of 6-8 nucleotides. The occurrence of seven of these elements in MALAT1 while other exosomal IncRNA such as HOTAIR and GAS5 contain only one partial sequence each, suggests that these elements are unique to certain IncRNAs such as MALAT1 and this facilitates its packaging into exosomes.

\section{Conclusions}

The packaging of MALAT1 into ADSC exosomes was regulated by ceramide synthase pathways. Overexpression of MALAT1 via ceramide synthase was important for cell migration in scratch assays. Since the inhibitor of sphingomyelin hydrolysis, GW4869, blocked MALAT1 transcription, packaging, secretion and cell migration, this suggests a pivotal role for ceramides in inclusion of IncRNA cargo in exosomes. GW4869 downregulation of MALAT1 exosomal content also resulted in the inhibition of mitochondrial ATP production, basal and maximal respiration. The fact that MALAT1 contains CRCE explains how ceramide regulates its transcription and packaging via ceramide dependent activities.

\section{Abbreviations}

ADSC: human adipose derived stem cells

Cer: ceramide

CM: conditioned media

CRCE: ceramide response cis-elements

sEV: small extracellular vesicle

HDF: human dermal fibroblasts

IncRNA: long non-coding RNA

MALAT1: metastasis-associated lung adenocarcinoma transcript 1

n-SMase: neutral sphingomyelinase

OCR: oxygen consumption rate

RBD: RNA binding domain

ROS: reactive oxygen species

\section{Declarations}


- This paper was funded by Dept of Veterans Affairs. The authors have no other financial involvement with any organization or entity with a financial interest in or financial conflict with the subject matter or materials discussed in the manuscript.

- Ethics approval and consent to participate: Not applicable.

- Consent for publication: All authors have consented to publication.

- Availability of data and material: All data and materials are available upon request.

- Competing Interests: There are no competing interests.

- Funding: Funded by the Department of Veterans Affairs, 1 |01BX0003689.

- Authors contributions: X.K. cultured the stem cells, isolated the exosomes and sEV and conducted the experiments reported. N.A.P. assisted in characterizing exosomes and in working with their isolation. C.E.C. collaborated with us on the presence of Ceramide response cis-elements in MALAT1, and the role of ceramide synthase pathways in exosome secretion and MALAT1 induction. D.R.C. planned the experiments, interpreted the data, and wrote the manuscript. All authors read and approved the final manuscript.

- Acknowledgements: None.

- Authors' information: Xaioyuan Kong (xkong@usf.edu), Niketa A.Patel (niketa@usf.edu), Charles E. Chalfant (cechalfant@usf.edu), Denise R. Cooper (dcooper@usf.edu).

\section{References}

1. Thery, C., Exosomes: secreted vesicles and intercellular communications. F1000 Biol Rep 2011, 3, 15.

2. Trajkovic, K.; Hsu, C.; Chiantia, S.; Rajendran, L.; Wenzel, D.; Wieland, F.; Schwille, P.; Brugger, B.; Simons, M., Ceramide triggers budding of exosome vesicles into multivesicular endosomes. Science 2008, 319 (5867), 1244-7.

3. Spinelli, C., Adnani, L., Choi, D. Janusz, R., Extracellular vesicles as conduits of non-coding RNA emission and intercellular transfer in brain tumors. Non-Coding RNA 2019, 5(1), 1-26.

4. Zhang, B.; Arun, G.; Mao, Y. S.; Lazar, Z.; Hung, G.; Bhattacharjee, G.; Xiao, X.; Booth, C. J.; Wu, J.; Zhang, C.; Spector, D. L., The IncRNA Malat1 is dispensable for mouse development but its transcription plays a cis-regulatory role in the adult. Cell Rep 2012, 2 (1), 111-23.d

5. Wilusz, J. E.; Freier, S. M.; Spector, D. L., 3' end processing of a long nuclear-retained noncoding RNA yields a tRNA-like cytoplasmic RNA. Cell 2008, 135 (5), 919-32.

6. Patel, R. S.; Carter, G.; El Bassit, G.; Patel, A. A.; Cooper, D. R.; Murr, M.; Patel, N. A., Adipose-derived stem cells from lean and obese humans show depot specific differences in their stem cell markers, exosome contents and senescence: role of protein kinase $\mathrm{C}$ delta (PKCdelta) in adipose stem cell niche. Stem Cell Investig 2016, 3, 2.

7. Cooper, D. R.; Wang, C.; Patel, R.; Trujillo, A.; Patel, N. A.; Prather, J.; Gould, L. J.; Wu, M. H., Human Adipose-Derived Stem Cell Conditioned Media and Exosomes Containing MALAT1 Promote Human 
Dermal Fibroblast Migration and Ischemic Wound Healing. Adv Wound Care (New Rochelle) 2018, 7 (9), 299-308.

8. Wang, J.; Su, L.; Chen, X.; Li, P.; Cai, Q.; Yu, B.; Liu, B.; Wu, W.; Zhu, Z., MALAT1 promotes cell proliferation in gastric cancer by recruiting SF2/ASF. Biomed Pharmacother 2014, 68 (5), 557-64.

9. Cooper, D. R., Wang, C., Patel, R., Trujillo, A., Patel, N.A., Prather, J., gould, L.J., Wu, M.H. Huan adiposederived stem cell conditioned media and exosomes containing MALAT1 promote human dermal fibroblast migration and ischemic wound healing. Adv Wound Care (New Rochelle), 2018,7:299-308.

10. Verderio, C.; Gabrielli, M.; Giussani, P., Role of sphingolipids in the biogenesis and biological activity of extracellular vesicles. J Lipid Res 2018, 59 (8), 1325-1340.

11. Massiello, A.; Salas, A.; Pinkerman, R. L.; Roddy, P.; Roesser, J. R.; Chalfant, C. E., Identification of two RNA cis-elements that function to regulate the $5^{\prime}$ splice site selection of $\mathrm{Bcl}-\mathrm{x}$ pre-mRNA in response to ceramide. J Biol Chem 2004, 279 (16), 15799-804.

12. Long, S. D.; Pekala, P. H., Lipid mediators of insulin resistance: ceramide signalling down-regulates GLUT4 gene transcription in 3T3-L1 adipocytes. Biochem J 1996, 319 (Pt 1), 179-84.

13. Teixeira, V.; Medeiros, T. C.; Vilaca, R.; Ferreira, J.; Moradas-Ferreira, P.; Costa, V., Ceramide signaling targets the PP2A-like protein phosphatase Sit4p to impair vacuolar function, vesicular trafficking and autophagy in Isc1p deficient cells. Biochim Biophys Acta 2016, 1861 (1), 21-33.

14. Dobrowsky, R. T.; Hannun, Y. A., Ceramide stimulates a cytosolic protein phosphatase. J Biol Chem 1992, $267(8)$, 5048-51.

15. Gulbins, E.; Kolesnick, R., Measurement of sphingomyelinase activity. Methods Enzymo/ 2000, 322, $382-8$.

16. Milhas, D.; Clarke, C. J.; Idkowiak-Baldys, J.; Canals, D.; Hannun, Y. A., Anterograde and retrograde transport of neutral sphingomyelinase-2 between the Golgi and the plasma membrane. Biochim Biophys Acta 2010, 1801 (12), 1361-74.

17. Dinkins, M. B.; Dasgupta, S.; Wang, G.; Zhu, G.; Bieberich, E., Exosome reduction in vivo is associated with lower amyloid plaque load in the 5XFAD mouse model of Alzheimer's disease. Neurobiol Aging 2014, 35 (8), 1792-800.

18. Hutchinson, J. N.; Ensminger, A. W.; Clemson, C. M.; Lynch, C. R.; Lawrence, J. B.; Chess, A., A screen for nuclear transcripts identifies two linked noncoding RNAs associated with SC35 splicing domains. BMC Genomics 2007, 8, 39.

19. Dominguez, D.; Freese, P.; Alexis, M. S.; Su, A.; Hochman, M.; Palden, T.; Bazile, C.; Lambert, N. J.; Van Nostrand, E. L.; Pratt, G. A.; Yeo, G. W.; Graveley, B. R.; Burge, C. B., Sequence, Structure, and Context Preferences of Human RNA Binding Proteins. Mol Cell 2018, 70 (5), 854-867 e9.

20. Elsherbini, A.; Bieberich, E., Ceramide and Exosomes: A Novel Target in Cancer Biology and Therapy. Adv Cancer Res 2018, 140, 121-154.

21. Hernandez-Corbacho, M. J.; Salama, M. F.; Canals, D.; Senkal, C. E.; Obeid, L. M., Sphingolipids in mitochondria. Biochim Biophys Acta Mol Cell Biol Lipids 2017, 1862 (1), 56-68. 
22. Janda, J.; Nfonsam, V.; Calienes, F.; Sligh, J. E.; Jandova, J., Modulation of ROS levels in fibroblasts by altering mitochondria regulates the process of wound healing. Arch Dermatol Res 2016, 308 (4), 239-48.

23. Kogot-Levin, A.; Saada, A., Ceramide and the mitochondrial respiratory chain. Biochimie 2014, 100, 88-94.

24. Zigdon, H.; Kogot-Levin, A.; Park, J. W.; Goldschmidt, R.; Kelly, S.; Merrill, A. H., Jr.; Scherz, A.; PewznerJung, Y.; Saada, A.; Futerman, A. H., Ablation of ceramide synthase 2 causes chronic oxidative stress due to disruption of the mitochondrial respiratory chain. J Biol Chem 2013, 288 (7), 4947-56.

25. Gudz, T. I.; Tserng, K. Y.; Hoppel, C. L., Direct inhibition of mitochondrial respiratory chain complex III by cell-permeable ceramide. J Biol Chem 1997, 272 (39), 24154-8.

26. Beckham, T. H.; Lu, P.; Jones, E. E.; Marrison, T.; Lewis, C. S.; Cheng, J. C.; Ramshesh, V. K.; Beeson, G.; Beeson, C. C.; Drake, R. R.; Bielawska, A.; Bielawski, J.; Szulc, Z. M.; Ogretmen, B.; Norris, J. S.; Liu, X., LCL124, a cationic analog of ceramide, selectively induces pancreatic cancer cell death by accumulating in mitochondria. J Pharmacol Exp Ther 2013, 344 (1), 167-78.

27. Zhao, Y., Cui, J., Hu, J., Hoffman, A.R., Effect of MALAT1 in the crosstalk between nucleus and nitochondria on mitochondrial reprogramming in hepatocellular carcinoma cells. Journal of Clinical Oncology 2019, 37.15 (Supple.e14711).

28. Zhao, Y., Liu, S., Zhou,L., Li, X., Meng, Y., Li, Y., Lingyu, L., Jiao, B., Bai, L., Yu, Y., Zhang, S., Li, W., Hoffman, A.R., Hu, J.F., Cui, J., Abberrant shuttling of long noncoding RNAs during the mitochondrianuclear crosstalk in hepatocellular carcinoma cells. Am J Cancer Res 2019, 9 (5), 999-1008.

\section{Tables}

Due to technical limitations, Tables 1 and 2 are only available as a download in the Supplemental Files section.

\section{Figures}




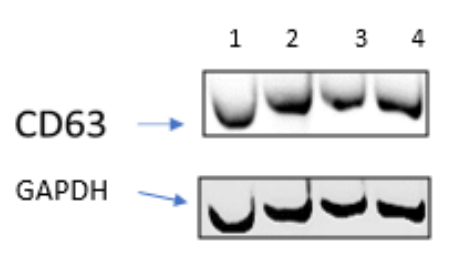

1.Control Exosomes 2.GW4869 Exosomes 3.C2 Exosomes 4.C6 Exosomes
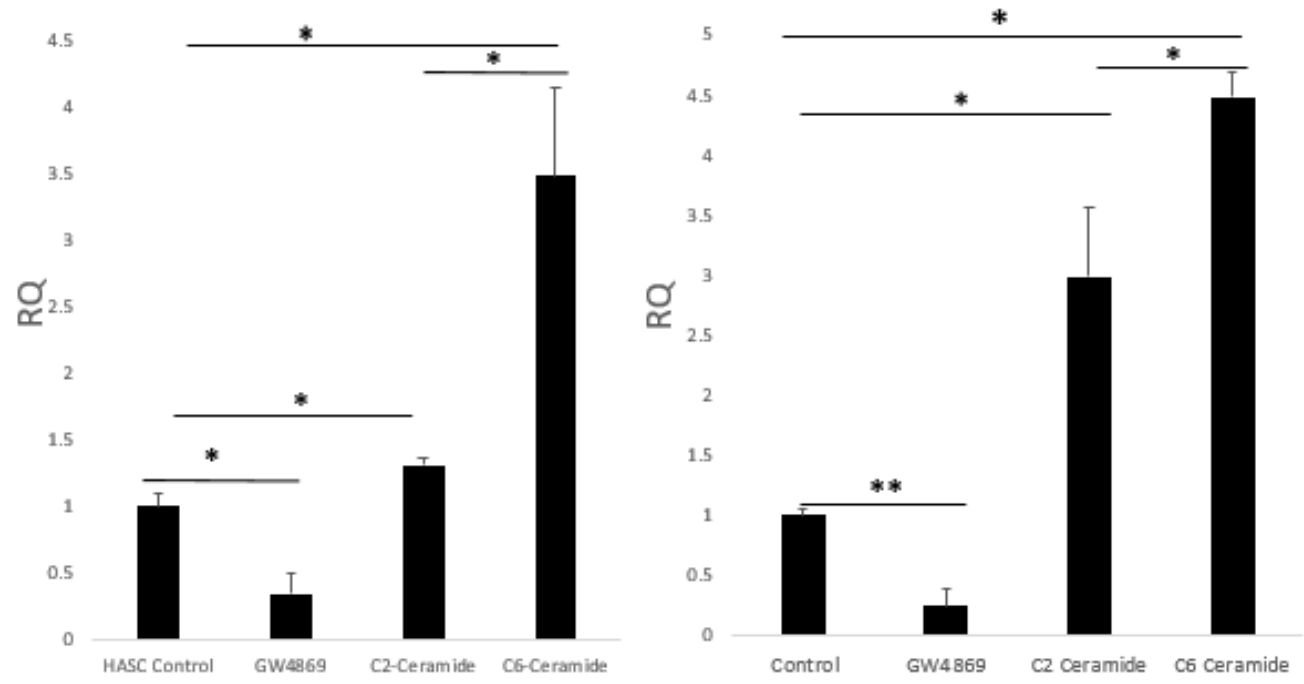

\section{Figure 1}

MALAT1 levels in hADSC and exosomes after treatment with modulators of ceramide synthesis. A. Expression of CD63 by exosomes from hADSC treated with modulators of ceramide synthesis. $\mathrm{B}$. hADSC were cultured and MALAT1 was measured in hADSC RNA after $48 \mathrm{hrs}$ in control cells, cells treated with GW4869, C2- and C6- ceramide. ${ }^{*} p<0.05$ as determined by Graph Pad Two-Way ANOVA. C. hADSC were cultured and treated with GW4869, C2- and C6ceramide and CM was collected after 48 hrs. Exosomes were isolated and MALAT1 expression was measured by RT-PCR. ${ }^{*} p<0.05$ and ${ }^{*} \mathrm{k}<0.01$ as determined by Graph Pad Two-Way ANOVA.

A Scratch Assay -Exosome Treated HDF-a Cells (10X Magnify)

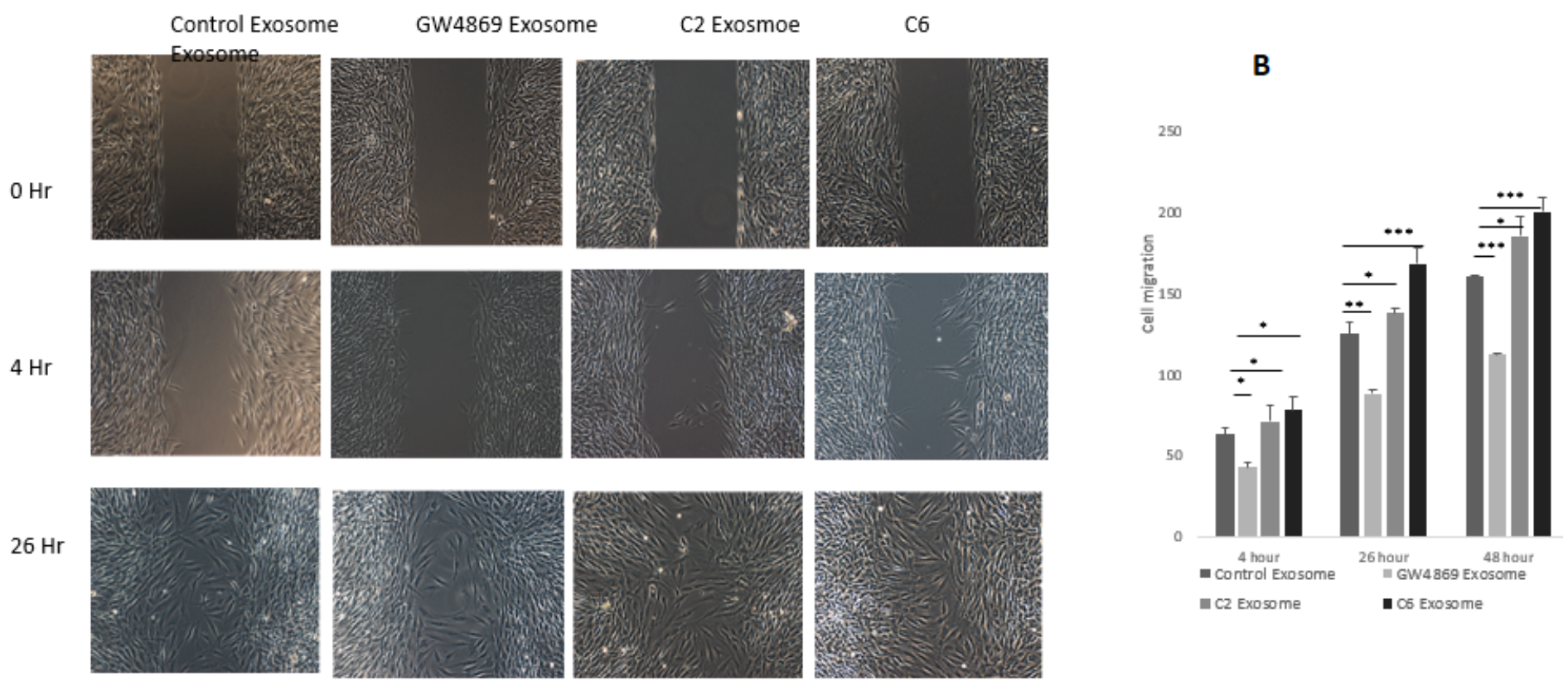


Cell migration assays using HDF-a treated with exosomes from cells as indicated. A. The cell free gap was created by Ibidi inserts, mitomycin C teated cells were incubated with $20 \mu \mathrm{g} / \mathrm{ml}$ exosomes and the images of cell migration were captured at $0,4,8 \mathrm{hr}$, and $26 \mathrm{hrs}$. B. Migrating cells were counted by Olympus CellSens software. Data are expressed as mean + SEM and was analyzed using Graph Pad Two Way ANOVA. ${ }^{*} p<0.05$ and ${ }^{* *} p<0.01$ were considered to be statistically significant compared to control exosomes.
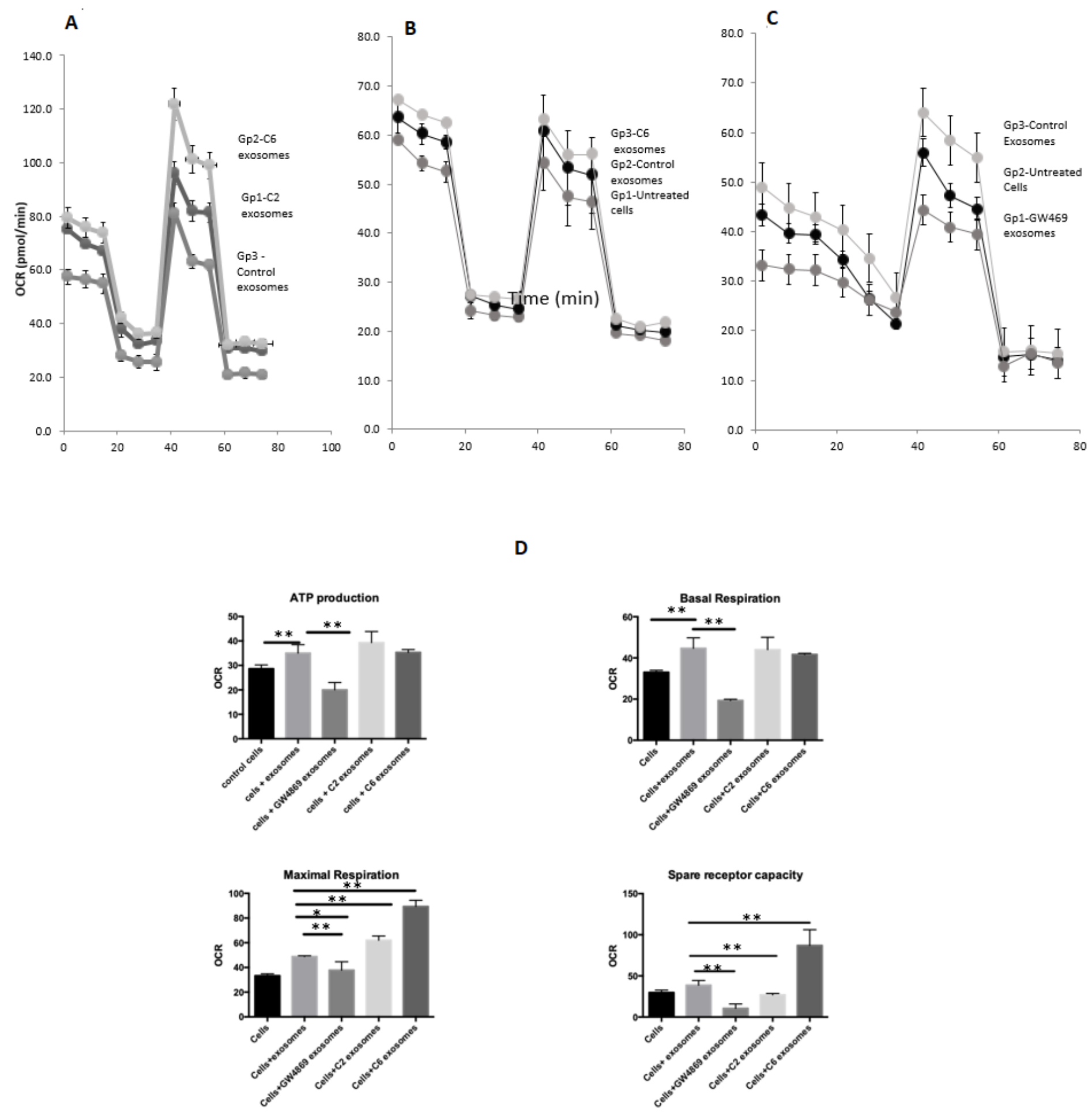

Figure 3 
Seahorse Mitocondrial Stress Test performed on HDF-a treated with exosome isolates. A. Mitochondrial respiration is indicated by oxygen consumption rates (OCR) was detected under the conditions of oligomycin $(1 \mu \mathrm{M}$, the uncoupler at $15 \mathrm{~min}), \mathrm{FCCP}(1 \mu \mathrm{M}$, at $35 \mathrm{~min})$ and the electron transport inhibitor rotenone $(0.5 \mu \mathrm{M}$, at $50 \mathrm{~min})$. Group 1 are untreated cells, Group 2 are cells treated with control exosomes, and Group 2 are cells treated with C6-exosomes. B. Group1 ar untreated cells, group2 are cells treated with control exosomes, and group 3 are cells treated with $\mathrm{C} 6$ exosomes. C. Group 1 are cells treated with GW4879 exosomes. Group 2 are untreated cells, and Group 3 are cells treated with control exosomes. Each panel is representative of one experiment run in duplicate. Experiments are run with 5800 cells per well and normalized to the cell number calculated after the end of the experiment. D. ATP production, Basal respiration, Maximal respiration, and Spare receptor capacity are compared between the control cells and cells treated with exosome isolates. ${ }^{*} \mathrm{p}<0.05$, and ${ }^{*} \mathrm{k}<0.01$ as determined by Student $\mathrm{t}$-test. $\mathrm{N}=4$ separate experiments run in duplicate. Experiments were normalized to cell number for data analysis.

\section{Supplementary Files}

This is a list of supplementary files associated with this preprint. Click to download.

- GagggaggcaggcgaTable1ref11.docx

- tableexosomesizewithceramide.pptm 